\title{
Genetic selection schemes - an important part of sustainable livestock improvement?
}

\author{
G. E. Pollott \\ Wye College, University of London, Ashford, Kent TN25 5AH
}

\section{Introduction}

The development of a sustainable livestock production system commonly involves the solution of a range of technical problems associated with animal health, nutrition/food supply and system management. These are often essential steps before any surplus animal products become available for sale, barter or to help increase living standards. The ability of a breeding programme to contribute to sustainable improvements in livestock performance is often overlooked. However, genetic gains are cumulative and will only disappear if genetically superior stock are removed from the population.

Attention to the genetic improvement of stock, if practised at all, is most commonly confined to breed substitution. The usual situation is where currently available breeds are thought to be poor producers, for the traits of interest, compared with other breeds either in other regions of the same country, or from other countries.

Breed substitution of this sort is based on the often erroneous assumption that no genotype environment interactions exists for the traits of economic value. Clearly, when genotypes are moved from an environment where their genetic value is known, to a similar environment, then they are likely to perform at a similar level. A good example of this is the importation of Holsteins into Britain from Canada and USA. However, there are many examples where such importations have clearly been less successful. This may not matter if the reduced performance in the new environment is still better than the performance from local stock but often the challenges of disease, climate and nutrition are such that performance is poor.

\section{Methods}

Within-population selection schemes

One of the options available to a livestock improvement programme is the use of withinpopulation selection of superior genotypes and the dissemination of these genotypes for wider use. Such schemes are well documented for many countries but depend on a combination of technical skills not widely found throughout the world. These include an understanding of quantitative genetics as applied to farmed livestock, a good appreciation of statistical genetics, advanced computer literacy and the requirements of an objective data handling system for recording livestock performance. Clearly training in all these areas is essential to run any modern livestock breeding programme.

\section{Components of a breeding programme}

A planned breeding programme has many components, all of which require particular skills. A list of these components is given below.

1. Define the breeding objectives of the programme and the environment of the target population.

2. Choose the selection criteria that will be measured to achieve the breeding objectives.

3. Consider the breeding methods and structures available to implement the selection criteria.

4. Set up and run a recording scheme to gather the required data.

5. Estimate the genetic parameters of the traits of interest.

6. Compute breeding values of the recorded population.

7. Compute performance indexes.

8. Rank and select the replacements from the recorded population.

Many of these stages require specialized computer programs and expertise in order to evaluate the animals and methods properly. The availability of these programs and expertise can be a severe limitation on the effective design and implementation of a within-population selection scheme.

\section{Availability of computer programs}

Genetic population modelling. No programs are available but spreadsheets and general modelling packages are useful. 
Genetic parameter estimation. Programs for estimating genetic parameters by the preferred method (Restricted Maximum Liklihood) are available but need a minicomputer and Fortran 77 to run them. Older methods (Least-squares) are available for microcomputers but suffer from several drawbacks.

Breding value estimation. Programs for estimating breeding values are available but the preferred method (Best Linear Unbiased Prediction) requires a minicomputer and Fortran 77.

Recording and data handling. No one program suits all requirements but database software for microcomputers is now very flexible and programmable.
Expertise in the use of these programs is more widely available due to the general nature of the software.

\section{Conclusions}

Once disease and nutrition have less of an impact on animal productivity, breeding programmes can be introduced to enhance the sustainability of performance improvements. Genetic improvement is technically demanding but has been shown to be effective in many situations. The development of local skills in the application of genetics to animal breeding, computing and recording is a necessary requirement for these schemes. In many situations a centre for breeding value and parameter estimation may be the best way to utilize a scarce commodity. 